\title{
La terminologie commune et les principes directeurs du droit des contrats
}

\section{Denis Mazeaud}

Published online: 29 August 2008

(C) ERA 2008

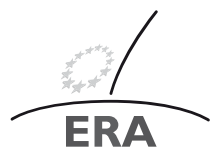

EUROPÄISCHE RECHTSAKADEMIE ACADEMY OF EUROPEAN LAW ACADEMIE DE DROIT EUROPEEN ACADEME DE DROT UROPEN TRIER - TREVES - TREYIR

\section{Introduction}

Je souhaiterais présenter le fruit de quatre années de travail réalisé par un groupe dont j'ai eu le plaisir, en compagnie de Mme Fauvarque-Cosson, de coordonner les travaux qui lui avaient été confiés au sein de notre réseau CoPECL.

Avant de commencer la présentation proprement dite de la terminologie commune et des principes directeurs du droit des contrats, j'aimerais, en guise d'introduction, revenir brièvement sur la situation finalement assez particulière du groupe que j'ai animé avec Mme Fauvarque-Cosson.

Situation assez particulière, d'abord, en raison du contexte français existant à l'époque de la création du réseau CoPECL. C'est peu dire, en effet, qu'une certaine hostilité à l'égard de l'idée même d'un droit européen des contrats existait, il y a quatre ans, au sein de la communauté juridique française. A tel point d'ailleurs que lors des célébrations du bicentenaire du code civil français, la question de la codification et de l'harmonisation européenne du droit privé en général et du droit des contrats en particulier fut au centre de la plupart des communications et des débats et même des controverses parfois extrêmement vifs. Et je me souviens même avoir vu le Premier Président de la Cour de cassation française quitter ostensiblement le grand salon de la Sorbonne pour protester contre les propos europhobes tenus par un célèbre professeur français. Tout cela pour dire qu'il n'a vraiment pas été facile à l'époque de convaincre les membres des deux associations, qui composent le groupe dont je vais vous exposer les travaux, de rejoindre le réseau CoPECL et de participer

Denis Mazeaud $(\bowtie)$

Professeur à l'Université Panthéon-Assas

92 rue d'Assas, 75006 Paris, France

e-mail: denis.mazeaud@wanadoo.fr

Cet article a été présenté lors d'un séminaire de 1'ERA portant sur «Le Projet de Cadre Commun de Référence», organisé en coopération avec le Joint Network on European Private Law les 6 et 7 mars 2008 à Trèves. 
à l'élaboration du cadre commun de référence. Et j'ai encore en mémoire l'ambiance pénible dans laquelle les assemblées générales de l'Association Henri Capitant et de la Société de législation comparée ont finalement, après des discussions assez dures, voté en faveur d'une participation à cette aventure contractuelle européenne. Mais sans vouloir jouer les héros ni les martyrs, il faut donc savoir que pour un juriste français, participer ouvertement à cette entreprise d'harmonisation européenne du droit privé, c'est s'exposer souvent à des critiques très déplaisantes, telle que de se faire traiter de «collaborateur» et risquer de se fâcher avec ceux des juristes français qui considèrent que le génie juridique français, et précisément le Code civil qui l'incarne, a tout à perdre dans cette aventure européenne et dans la construction d'une Europe juridique.

Et je suis certain que Christian von Bar se souvient de l'ambiance assez curieuse dans laquelle nous l'avons rencontré, il y a quatre ans à Paris, pour évoquer avec lui, lors d'un déjeuner qui réunissait le président de l'Association Henri Capitant, Michel Grimaldi, Bénédicte Fauvarque-Cosson, au nom de la Société de législation comparée, les missions confiées à notre groupe au sein du réseau. Nous étions réunis, comme des conspirateurs, dans l'arrière salle d'un restaurant du quartier latin à l'abri des regards indiscrets et quand, à la fin du repas, vous nous avez demandé de visiter les locaux de la Faculté de droit au Panthéon, nous avons inventé un prétexte pour trouver un autre lieu de promenade. En réalité, vous le comprenez maintenant Cher Christian, nous voulions assurer votre sécurité ... Sérieusement, lors de ce déjeuner, nous avions évoqué les missions dont le groupe «Association Capitant/Société de législation comparée» (ci-après «AHC/SLC») était officiellement chargé au sein du réseau CoPECL. Et sur ce point précis, notre groupe se trouve une nouvelle fois dans une situation particulière.

En effet, à l'origine notre groupe était un groupe d'évaluation chargé précisément d'évaluer les travaux des groupes chargés de la rédaction du projet de cadre commun de référence. Or, pendant ces quatre dernières années, le fait est que nous n'avons effectué aucun travail d'évaluation à proprement parler, faute d'avoir reçu de texte à évaluer ... Mais, peu importe au fond puisque, dès l'origine de la constitution du réseau, des missions plus larges que la simple évaluation des travaux des autres groupes nous avaient été confiées. D'une part, il nous avait été demandé, de façon officielle, de préparer les deux premiers volets du cadre commun de référence, c'est-à-dire concrètement de rédiger «des Principes fondamentaux communs du droit européen des contrats» ainsi qu'une terminologie contractuelle commune. D'autre part, vous vous souvenez sans doute, Cher Christian, que lors de notre déjeuner d'il y a quatre ans vous nous avez confié officieusement et finalement assez imprudemment une autre mission. Vous nous aviez fait remarquer, dans l'euphorie suscitée par la convivialité de notre accueil et d'une bonne bouteille de Bordeaux, que si nous nous limitions à la rédaction de principes directeurs et d'une terminologie commune, nos travaux risquaient d'être exclusivement théoriques et dogmatiques, et par conséquent de ne pas peser très lourd dans la réalisation du cadre commun de référence. Et vous nous avez alors incités et invités à nous investir aussi dans le troisième volet du cadre commun de référence, à savoir l'élaboration «des règles modèles, formant le corps $d u C C R$ ». Plus précisément, vous nous avez conseillés, afin que les travaux de notre groupe soient véritablement utiles dans la perspective de l'élaboration du CCR à préparer des contre-projets, des projets alternatifs de révision des Principes 
du droit européen du contrat et de droit de la responsabilité civile. Honnêtement je suis aujourd'hui persuadé que lorsque vous nous avez fait ces propositions, vous ne vous faisiez pas la moindre illusion sur l'avenir des travaux de notre groupe et que, dans l'avion qui vous ramenait vers Osnabrück, vous pensiez que la concurrence que vous aviez ainsi très opportunément initiée au sein de notre réseau ne s'exercerait pas, faute de ... concurrents. Vous avez, en effet, fréquenté suffisamment de professeurs français au sein du Study Group pour savoir que, à quelques exceptions près évidemment qui se trouvent d'ailleurs dans cette salle mais par sur cette estrade, ils sont en général plus doués pour faire des promesses que pour les réaliser. Mais s'est produit ce que l'on pourrait appeler le miracle français, c'est-à-dire un événement inattendu, invraisemblable, incroyable: le groupe AHC/SLC a travaillé pendant ces quatre années! Non seulement, il a rempli les missions officielles qui lui avaient été confiées au sein du réseau CoPECL, puisque sont d'ores et déjà publiés deux ouvrages qui contiennent la «Terminologie contractuelle commune» et les Principes directeurs du droit européen des contrats. Mais encore, notre groupe a rempli une partie de la mission qui lui avait été officieusement confiée, puisqu'un projet de révision des Principes du droit européen du contrat a été rédigé et fait lui aussi l'objet d'une publication conjointe avec les Principes directeurs du droit européen du contrat, dans un ouvrage intitulé «Principes contractuels communs».

Et paradoxalement, alors, la situation particulière de notre groupe au sein du réseau CoPECL est encore plus accentuée, plus forte, et vous me permettrez ici de ne pas utiliser la langue de bois et de parler très franchement de cette situation assez embarrassante pour notre groupe. En effet, nous avons le sentiment assez net aujourd'hui que finalement notre groupe et les travaux qu'il a réalisés sont accueillis avec une certaine méfiance, avec une certaine réserve et que finalement nous sommes quelque peu marginalisés au sein du réseau, à tel point, et pardonnez-moi une fois encore ma franchise, qu'aujourd'hui je me demande sérieusement si nous n'avons pas travaillé pour rien ou presque rien. Je reviendrai plus tard dans cette intervention sur ces questions en évoquant l'avenir de nos travaux, mais je voudrais d'ores et déjà retenir deux illustrations anecdotiques mais significatives du particularisme de notre groupe au sein du réseau et des réactions suscitées par nos travaux au sein du réseau.

La première tient aux difficultés que nous avons rencontrés pour publier nos travaux en langue anglaise. L'éditeur que nous avons sollicité craignait en effet qu'une telle publication subisse un échec commercial à cause de la prétendue perspective française dans laquelle nos travaux ont été conçus. Sur ce point, je tiens à être extrêmement clair. Certes, le groupe AHC/SLC était essentiellement composé d'universitaires français et a, pour cette raison, travaillé en langue française, mais c'est le souffle de l'Europe qui a animé la rédaction de nos travaux. En clair, il n'a jamais été question pour notre groupe de faire acte de résistance nationale en défendant coûte que coûte le génie contractuel français contre le reste de l'Europe contractuelle. Certes, l'existence de notre groupe s'explique par notre volonté d'assurer une présence française dans ce réseau européen, mais nos travaux s'inscrivent dans une perspective d'harmonisation européenne et certainement pas dans l'idée de défendre les exceptions contractuelles françaises. Ole Lando, qui s'était étonné, en commentant l'Avant-projet de réforme du droit des obligations et de la prescription qu'en droit français certains mythes ont la peau dure, constatera que dans le projet de révision des Principes du droit européen du contrat que nous avons élaboré, nous 
n'avons pas sacrifié à la mythologie contractuelle française. Entre autres exemples, et dût-il nous en coûter, nous n'avons pas réintégré la notion de cause dans ces Principes, en revanche nous avons maintenu les règles qui permettent la nullité unilatérale du contrat pour lésion qualifiée (profit excessif ou avantage déloyal) et la révision judiciaire du contrat pour imprévision. Autant dire que nous n'avons pas été dans nos travaux animés par une sorte de nostalgie contractuelle française, bien au contraire.

La seconde illustration de la situation extrêmement particulière de notre groupe au sein du réseau CoPECL procède de la présentation même du programme du colloque qui nous réunit aujourd'hui. Je devine que vous allez penser que décidément les français sont d'incorrigibles amoureux de la forme et du plan ordonné et structuré, mais avouez que mon intervention est programmée à une place quand même assez curieuse de cette première journée. Celle-ci est en effet divisée en trois parties: 1) le réseau CoPECL; 2) Droit des contrats et valeurs sous jacentes; 3) Le projet de cadre commun de référence. Objectivement, du moins c'est ce que je pense, la présentation de la terminologie commune et des principes directeurs du droit des contrats aurait pu légitimement être intégrée sinon dans la présentation générale du projet de cadre commun de référence, puisque sur le droit des contrats nous avons proposé un projet alternatif, du moins dans la partie de la journée consacrée au «Droit des contrats et valeurs sous-jacentes» tant il est vrai que les principes directeurs du droit des contrats que nous avons rédigés sont irréductiblement liés à la question des valeurs qui doivent irriguer et animer le droit européen des contrats. Mais au lieu de cela, ma présentation est intégrée dans la partie de la journée intitulée «Le réseau CoPECL»... Les optimistes me diront que je devrais me réjouir car cela signifie que Hans SchulteNölke est vraiment très indulgent avec notre groupe qui depuis des mois et des mois l'ennuie avec ses états d'âmes et ses revendications incessantes ... Les pessimistes, et notamment les nombreux juristes français qui nous ont très sévèrement critiqués pour avoir rejoint ce réseau et pour avoir accepté de participer à la réalisation du cadre commun de référence, me diront que notre groupe a servi de caution française à une entreprise européenne qui en avait seulement besoin pour asseoir sa légitimité, en somme notre groupe aurait simplement eu une dimension diplomatique mais aucun rôle scientifique et politique réel ...

L'avenir nous dira si, oui ou non, nous ne sommes effectivement qu'un groupe virtuel ou si le cadre commun de référence sera effectivement composé des travaux de tous les groupes du réseau constitué pour l'élaborer.

En attendant, et après cette trop longue introduction, il est plus que temps de vous présenter les travaux de notre groupe comme le prévoit notre programme. Dans cette perspective, je m'arrêterai successivement sur la méthode, le domaine, la structure, l'esprit et la substance des travaux en questions.

\section{La méthode}

Qu'il s'agisse de la terminologie contractuelle commune, des Principes directeurs du droit européen des contrats ou de notre projet de révision des principes directeurs du droit européen du contrat, tous ces travaux ont sur le plan de la méthode un dénominateur commun qui réside dans la comparaison. C'est en effet la méthode 
comparative qui a constitué le fil directeur de la préparation et de la réalisation de nos différents travaux. Cette unité dans la méthode a été rendue possible par le fait que si l'élaboration de chacun de ces travaux a été confiée à des groupes différents, la coordination de tous ces groupes, en particulier en ce qui concerne l'unité de leurs méthodes de travail, a été assurée par les deux coordonnateurs du groupe AHC/SLC, à savoir Bénédicte Fauvarque-Causson et moi-même.

Concrètement et précisément, en ce qui concerne, en premier lieu, la terminologie contractuelle commune, les rédacteurs du groupe, dont faisaient partie deux chercheurs allemand et italien, ont exploités pour les besoins de leurs travaux:

- d'abord le droit romain, les droits positifs nationaux (allemand, américain, anglais, belge, français, italien, luxembourgeois, néerlandais, québécois, russe et suisse), les projets nationaux de réforme (Avant projet français de réforme du droit des obligations et de la prescription);

- ensuite, l'acquis communautaire entendu comme l'ensemble des traités, règlements, directives et conventions conclu dans le cadre de l'Union européenne et relatifs au droit des contrats;

- en outre, les conventions européennes, telles la $\mathrm{CEDH}$, et les codifications européennes doctrinales (PECL; Avant projet de code européen des contrats de l'Académie des privatistes européens; Draft common frame of reference);

- enfin, l'acquis international, à savoir les conventions internationales relatives au droit des contrats (CIVM, convention CNUDCI sur les garanties et lettres de crédit, convention de Rome sur la détermination de la loi applicable aux obligations contractuelles, convention des Nations Unies sur les lettres de change et les billets à ordre internationaux, Incoterms), les codifications savantes (Principes Unidroit).

Ce sont des matériaux de droit comparé à peu près identiques qu'ont exploité les deux groupes respectivement chargés de la rédaction des Principes directeurs du droit européen du contrat et de la révision des Principes du droit européen du contrat. En d'autres termes, chacun de ces groupes est parti d'une très vaste exploration de l'acquis national (droit positif et prospectif des pays membres de l'Union européenne entre autres, puisque les droits américains, québécois et suisse ont aussi été étudiés), de l'acquis communautaire (traités fondateurs et droit dérivé) et européen (conventions et codifications savantes), et de l'acquis international pour mener à bien les différents travaux qui leur avaient été confiés.

Pour en terminer avec ce discours sur la méthode qui a présidé à l'élaboration des travaux du groupe français, j'ajouterai qu'outre l'unité scientifique assurée en son sein par l'utilisation de la méthode comparative, une certaine cohésion a été recherchée dans la mesure où il nous est apparu que tous les travaux qui nous avaient été confiés étaient unis par un lien très fort. Ainsi, il est clair que la rédaction de principes directeurs du droit européen des contrats supposait pour le groupe qui en était chargé une réflexion commune avec ceux qui préparaient un projet de révision des PECL. De même, ceux qui travaillaient sur l'élaboration d'une terminologie contractuelle commune devaient nécessairement confronter leurs propres réflexions et recherches avec les rédacteurs des principes contractuels communs. Aussi, trois réunions communes étaient assurées tous les ans au sein de notre groupe, grâce auxquelles nous avons, je l'espère en tous les cas, réalisé un travail d'ensemble à la fois cohérent et harmonieux. 


\section{Le domaine}

Sur ce point, je serai extrêmement rapide puisque, mais c'est une différence sensible avec l'option retenue par les groupes qui ont rédigé le Draft Common Frame of Reference, notre groupe a choisi pour sa part de consacrer ses travaux au seul droit européen des contrats, conformément d'ailleurs aux directives tracées par la Commission elle-même dans les différentes communications dans lesquelles elle a envisagé l'élaboration d'un cadre commun de référence.

En particulier, ce domaine spécifique ressort nettement de la Communication du 12 février 2003, intitulée «Un droit européen des contrats plus cohérent. Un plan d'action», dans laquelle la Commission européenne avait initié le projet de cadre commun de référence.

A la vérité, si notre groupe n'a pas donné un plus large domaine à ses travaux, notamment si nous n'avons finalement pas, comme Christian von Bar nous y avait invités, rédigé un projet concurrent en matière de droit européen de la responsabilité civile, c'est essentiellement par manque de temps et de moyens, beaucoup plus qu'à la suite d'un choix réfléchi et délibéré.

\section{La structure}

Quelques mots maintenant sur la structure de nos travaux. A cet égard, j'insisterai en premier lieu sur leur homogénéité, à savoir qu'ils comportent tous des notes relatives aux instruments exploités pour les besoins de la comparaison entreprise, et des commentaires sur les textes anciens révisés et les textes nouveaux proposés.

En ce qui concerne plus particulièrement le projet de révision des Principes du droit européen du contrat, je préciserai simplement que, par rapport au Draft Common Frame of Reference, notre groupe est resté fidèle, sinon à la lettre du moins à l'esprit du plan initial retenu par la commission Lando. Certes quelques modifications ont été effectuées ${ }^{1}$ mais, comme dans la version initiale, le plan est articulé de façon chronologique autour des différentes étapes du processus contractuel.

Cette fidélité au plan initial se justifie par l'idée que nous avons constamment eue à l'esprit lors de la rédaction de nos travaux et selon laquelle le droit est avant tout fait pour les justiciables et non pour les juristes. Par conséquent, il importe que le plan soit intelligible et accessible, non seulement pour le juriste initié ou spécialiste de droit des contrats, mais aussi, mais surtout pour le consommateur de droit ou son conseil qui auront besoin de consulter ces règles contractuelles. Or, il nous a semblé que, dans leur état actuel, les Principes du droit européen du contrat cultivent ces deux vertus indispensables à l'effectivité et à la légitimité du droit que sont l'intelligibilité et l'accessibilité.

Je n'aurai évidemment pas l'arrogance d'affirmer ici que notre projet de révision est doté des mêmes qualités, mais j'oserai en revanche émettre à propos du plan

\footnotetext{
1) Ainsi, entre autres les Chapitres 4 (validité) et 15 (illicéité) initiaux ont été fusionnés au sein d'un seul chapitre 4, désormais intitulé «Invalidité»; le Chapitre 6 (contenu et effets) a été scindé en deux chapitres traitant séparément ces deux questions; le Chapitre 8 sur l'exécution accueille en son sein le Chapitre 16 relatif aux conditions.
} 
retenu dans le Draft Common Frame of Reference une opinion dissidente. Opinion dissidente car je sais que le plan retenu, qui conduit à éclater les règles contractuelles dans deux livres différents ${ }^{2}$, a été adopté à la majorité par le Coordinating committee de notre réseau. Mais pour ma part, pour avoir déjà utilisé le Draft Common Frame of Reference, je trouve que sa structure est complexe et beaucoup plus difficilement accessible pour les profanes du droit des contrats, notamment les jeunes chercheurs européens, et je pense qu'on a pas eu forcément raison de vouloir substituer la complexité à la simplicité, l'abstraction à la clarté et le droit savant au droit vivant.

\section{L’esprit}

A propos de l'esprit qui a animé nos différents travaux, je ne reviendrai pas sur le fait qu'il n'a jamais été question pour aucun membre de notre groupe d'exploiter les missions qui nous ont été confiées au sein de ce réseau pour faire acte de résistance, active ou passive, contre l'élaboration d'un instrument destiné à devenir le ferment d'un droit européen des contrats. Nous avons résolument joué le jeu de cette aventure contractuelle européenne en partant du principe que pour celle-ci soit menée à bien, autrement dit pour que cette entreprise visant à l'édification d'un modèle contractuel européen soit à la fois légitime et réussie, il était indispensable qu'elle intègre la diversité des droits contractuels nationaux, qu'elle se nourrisse de la richesse inhérente à cette diversité, qu'elle concilie les différences et non qu'elle les supprime au nom d'une uniformité aussi artificielle que vaine. Au fond, il nous a semblé indispensable d'avoir à l'esprit que ce modèle contractuel européen qu'il s'agissait de façonner soit pluriel, qu'il puise à plusieurs sources, qu'il soit irrigué par une pluralité de valeurs, d'impératifs et d'idées forces.

C'est dans cette perspective qu'a, en premier lieu, été élaborée la terminologie contractuelle commune. Le travail réalisé n'avait pas pour objectif de créer un simple lexique, ni à l'inverse une terminologie dogmatique qui s'imposerait dans la perspective de l'utilisation du cadre commun de référence. Le groupe s'est plutôt employé, en partant de la terminologie existante et déjà connue, à identifier les difficultés liés à l'emploi de certains termes consacrés dans les différents droits nationaux, règlements et directives communautaires, conventions et codifications européennes et internationales, puis à proposer des explications sur la terminologie exploitée dans ces différents instruments contractuels. Au fond, l'objectif poursuivi a été de mettre en lumière les principales divergences et convergences d'ordre terminologique décelées grâce à l'analyse comparative effectuée, et à détecter les difficultés qui peuvent intervenir lors de la traduction de tel ou tel terme spécifique. En définitive, on ne trouve point dans cette terminologie de définitions rigides des notions étudiées mais des lignes directrices d'analyse expliquant la diversité en matière de terminologie contractuelle et proposant non pas de la supprimer, ce qui est une mission impossible, mais de la comprendre et de la maîtriser dans la perspective de la rédaction définitive du cadre commun de référence.

\footnotetext{
2) Le livre II est traite des contrats et autres actes juridiques, le livre III est consacré aux obligations et
} droits correspondants, indépendamment de leurs sources. 
C'est exactement dans ce même esprit, consistant à proposer un modèle contractuel européen qui soit pluriel, c'est-à-dire qui reflète et qui respecte la diversité contractuelle européenne qu'ont été élaborés les principes directeurs du droit européen du contrat, ainsi que le projet de révision des Principes du droit européen du contrat, étant précisé que les deux sont irréductiblement liés. En effet, les Principes directeurs du droit européen des contrats constituent la matrice philosophique, le moule politique et le socle idéologique dont, en toute logique et en toute cohérence, les règles modèles du cadre commun de référence doivent nécessairement s'inspirer et fatalement constituer de simples applications spécifiques.

Raison pour laquelle il nous a semblé que ces Principes contractuels communs devaient, à l'image de ceux qu'avait en son temps rédigés la commission Lando, concilier les différentes idéologies contractuelles que sont le libéralisme et la justice sociale et reposer sur les différents impératifs de politique contractuelle que sont la liberté, la sécurité et la loyauté. Etant entendu que dans cette perspective, il nous a paru nécessaire que ces impératifs devaient être appréhendés sous l'angle de la complémentarité et pas sous celui de la contradiction. En clair, à tort ou à raison, mais c'est véritablement l'esprit qui a animé la réalisation de nos travaux, il nous a semblé que le modèle contractuel européen pour être un modèle viable et attractif devait être un modèle idéologiquement et politiquement métissé, et que dans cette perspective, il devait refléter une vision libérale du contrat et de son droit, mais aussi une vision morale et une vision sociale.

\section{La substance}

Désormais dévoilé l'esprit qui a animé nos différents travaux, je voudrais dire maintenant quelques mots sur leur substance, étant évidemment entendu que je n'aurai aucun souci d'exhaustivité dans ce domaine, car si je voulais les exposer in extenso, cela nous obligerait à ne pas déjeuner, ni dîner aujourd'hui et je pense que vous m'en tiendriez alors légitimement rigueur ... Aussi, vais-je simplement ici évoquer quelques aspects du contenu des trois volets du travail que notre groupe a accompli ces dernières années.

En premier lieu, pour ce qui concerne la terminologie contractuelle commune, je préciserai que les études réalisées portent sur les notions de «contrat», «obligation et devoir», «acte/fait juridique», «ordre public et règles impératives», «bonne foi», «faute et manquement», «préjudice», «dommages et intérêts» et «anéantissement».

Sans entrer dans les très riches détails de tous les enseignements que ces différentes études comportent, celles-ci révèlent, entre autres, parfaitement la dualité de la notion de contrat en Europe, contrat promesse dont le centre de gravité réside dans la volonté exprimée dans certains systèmes contractuels et contrat échange dans d'autres systèmes dont la pierre angulaire réside dans la confiance suscitée par la promesse faite ou le comportement adopté. Elles montrent les confusions et les approximations qui règnent dans l'utilisation des concepts d'ordre public et de règles impératives et leur incapacité à appréhender les principes fondamentaux visés par la $\mathrm{CEDH}$. Ces études conduisent aussi à s'interroger sur la légitimité et l'opportunité des distinctions conceptuelles qui existent à l'intérieur même de la notion de bonne foi, ainsi que sur la dimension fonctionnelle de ce concept dont on sait qu'il est 
souvent controversé si ce n'est dans son principe, du moins dans la portée qu'il est opportun de lui accorder. Enfin, mais cet inventaire est évidemment extrêmement partiel, ces travaux nous révèlent la grande diversité terminologique et conceptuelle qui règne en Europe à propos de la question de l'anéantissement du contrat et livrent de stimulantes propositions tendant à simplifier et à clarifier ce domaine sensible en raison du contentieux qu'il engendre.

En deuxième lieu, quant au contenu des Principes directeurs du droit européen des contrats, on indiquera qu'ils se déclinent autour des trois grands principes que sont la liberté, la sécurité et la loyauté contractuelle. Principes qui irradient, non seulement, les différents droits contractuels nationaux des pays de l'Union européenne, avec évidemment une intensité différente, mais aussi les codifications européennes et internationales que sont les Principes directeurs du contrat dans leur version initiale, l'Avant-projet de code européen des contrats préparé sous l'autorité du Professeur Gandolfi et les Principes d'Unidroit. Encore une fois, permettez-moi d'insister sur ce point, il nous a semblé que la légitimité, l'attractivité et la pérennité du modèle contractuel européen supposait qu'il ne soit pas le fruit d'une pensée unique, en clair qu'il ne soit pas exclusivement le fruit d'une idéologie libérale, mais au contraire qu'il ait plusieurs sources d'inspiration, concrètement qu'outre le courant libéral, il soit aussi irrigué par l'idéal de justice sociale et imprégné d'humanisme contractuel.

A partir de ce postulat, les valeurs fondatrices du modèle contractuel européen tel que nous l'avons conçu ont été déclinées à partir des principes de liberté, de sécurité et de loyauté contractuelles qui ont pour vocation de guider l'application et l'interprétation des règles contractuelles modèles, ainsi éventuellement que de fournir au juge des principes de solution dans les cas où aucune règle modèle appropriée n'aurait été prévue pour régler une question spécifique. Concrètement, la liberté contractuelle, et donc la dimension libérale qui innerve ces principes directeurs se décline précisément en trois principes que sont d'abord, la liberté des parties de conclure le contrat, le respect de la liberté et des droits des tiers et la liberté des parties de modifier ou de mettre un terme au contrat. Quant à la sécurité contractuelle, deuxième pilier sur lequel repose le modèle contractuel européen et qui lui confère une dimension morale, elle englobe le principe de la force obligatoire du contrat, les devoirs et les droits des tiers et l'idée de faveur pour le contrat. Enfin, l'impératif de loyauté contractuelle se décline en plusieurs principes, qui dotent le modèle contractuel européen d'une dimension sociale, et qui se traduisent concrètement par des devoirs de bonne foi, de collaboration et de cohérence.

Je passerai rapidement, en troisième lieu, sur la substance du projet de révision des principes du droit européen du contrat. D'une part, parce qu'ils reflètent logiquement pour l'essentiel les principes directeurs susvisés dont ils constituent des application spécifiques. D'autre part, parce que je ne souhaite pas empiéter sur les plates bandes de ma collègue Mme Fauvarque-Cosson qui doit évoquer la question de la révision des PECL et qui traitera donc probablement aussi du contenu de notre propre projet de révision.

Je préciserai simplement que notre projet de révision reste fidèle pour l'essentiel à l'esprit qui animait la version initiale des principes du droit européen du contrat telle qu'elle avait été conçue par la Commission Lando, même si nous proposons certaines modifications, certaines suppressions et certains ajouts. La raison pour laquelle il nous a semblé de bonne politique de rester fidèle aux grandes options politiques 
et techniques des Principes Lando est simple. Il nous est apparu qu'avec le temps, ces Principes avaient acquis une très forte légitimité et une très grande autorité dans la communauté juridique européenne. Résultats d'autant plus remarquables que la notoriété et le succès de ce best seller contractuel sont le résultat du processus le plus démocratique qui soit, puisqu'ils résultent de la seule qualité de ces Principes, sans qu'aucune intervention d'ordre institutionnel ou technocratique n'ait été nécessaire. Dès lors, comme disent les sportifs, pourquoi changer une équipe qui gagne? Pourquoi modifier des Principes qui ont fait leur preuve et que la communauté des juristes européens, mais aussi les juristes au-delà des frontières de la vieille Europe, ont, avec le temps, apprécié, adopté et intégrés dans leurs ouvrages, dans leurs enseignements et dans leurs pratiques?

Fidélité ne rime pourtant pas avec servilité évidemment et tout en demeurant proches des Principes dans leur version initiale, notre groupe a apporté quelques modifications de fond dans son projet de révision. Entre autres, et sans souci d'exhaustivité, dans le souci de favoriser le déploiement de la liberté contractuelle, nous avons ajouté une règle aux termes de laquelle une clause de répartition des risques de l'imprévision peut faire échec à la renégociation du contrat ordonnée par le juge. Pour renforcer la sécurité contractuelle, nous avons intégré dans les Principes de nouvelles règles relatives aux effets du contrat et à la durée des contrats renouvelés, prorogés ou tacitement reconduits. Enfin, pour garantir le respect de la loyauté contractuelle, nous avons encadré plus rigoureusement la résolution unilatérale du contrat et prévu que tout contrat, même conclu à durée indéterminée, aurait une durée minimum afin de protéger les intérêts légitimes du contractant ayant effectué des investissements lourds dans la perspective de la conclusion et de l'exécution du contrat.

\section{Conclusion}

En guise de conclusion, je souhaite dire quelques mots sur l'avenir des travaux que je viens de vous présenter rapidement. Je crois que l'avenir des travaux réalisés par le groupe «Association Capitant/Société de législation comparée» peut donner lieu à deux versions radicalement différentes.

La première version est pessimiste et signifierait que notre travail a été accompli en pure perte. En clair, nos travaux ne seraient absolument pas pris en considération dans le Cadre Commun de Référence qui sera remis dans quelques mois à la Commission. Le seul souvenir qui serait conservé de notre participation à ce réseau serait les livres qui sont d'ores et déjà publiés en langue française et qui vont être réunis dans un ouvrage en version anglaise. Cet avenir limité à une simple perspective académique et scientifique constituerait évidemment un échec cuisant pour notre groupe et une victoire retentissante pour tous ceux qui en France nous avaient fortement déconseillés de participer à cette aventure contractuelle européenne.

La seconde version est optimiste. Nos travaux pourraient jouer alors un double rôle dans la perspective de l'élaboration définitive du Cadre Commun de Référence. D'abord, la terminologie contractuelle commune pourrait être intégrée dans les documents destinés à être joints aux règles modèles. Ensuite, les Principes directeurs du droit européen des contrats devraient être intégrés dans le Livre I du Draft Common Frame of Reference lequel ne comporte, dans sa version actuelle, aucune disposition 
de ce genre. Ceci nous semble s'imposer car il nous paraît pour tout dire assez invraisemblable et surtout extrêmement dommageable qu'un ouvrage qui contiendra les règles qui composeront le modèle contractuel européen ne comportent aucune disposition générale susceptible d'identifier la philosophie, les valeurs fondamentales et les impératifs politiques qui irriguent et fondent les règles modèles. Au fond, il est important que le modèle contractuel européen qui circulera demain ne soit pas simplement une compilation de règles et que le socle, les piliers, les fondations sur lesquelles les dites règles reposent soient clairement exprimés et identifiés. Enfin, en ce qui concerne notre propre projet de révision des Principes du droit européen du contrat, pourquoi ne pas jouer le jeu dans les mois qui viennent d'une véritable mise en concurrence avec le Draft Common Frame of Reference, tout du moins avec les livres II et III qui sont relatifs au contrat? Pourquoi ne pas considérer qu'il existe aujourd'hui sur le marché du droit européen du contrat deux offres concurrentes qu'il convient de comparer dans la perspective de l'élaboration définitive du Cadre Commun de Référence? Pourquoi ne pas organiser dans quelques mois un colloque qui s'intitulerait «Droit européen des contrats: deux versions de l'avenir»? Je vous fais ce matin cette offre. L'avenir nous dira si la concurrence libre et loyale tente certains d'entre vous ... 